\title{
Chiral Current in a Graphene Battery
}

\author{
Hector Torres-Silva ${ }^{1}$, Diego Torres Cabezas ${ }^{2}$ \\ ${ }^{1}$ Escuela de Ingeniería Eléctrica Electrónica, Universidad de Tarapacá, Arica, Chile; ${ }^{2}$ Modernización y Gobierno Electrónico, Mi- \\ nisterio Secretaría General de la Presidencia, Santiago, Chile. \\ Email: htorres@uta.cl
}

Received August $2^{\text {nd }}, 2012$; revised September $5^{\text {th }}, 2012$; accepted September $15^{\text {th }}, 2012$

\begin{abstract}
We review the formulation of graphene's massless Dirac equation, under the chiral electromagnetism approach, hopefully demystifying the material's unusual chiral, relativistic, effective theory. In Dirac's theory, many authors replace the speed of light by the Fermi velocity, in this paper we deduce that in graphene the Fermi velocity is obtained from the connection between the electromagnetic chirality and the fine structure constant when the electric wave $\boldsymbol{E}$ is quasi parallel to the magnetic wave $\boldsymbol{H}$. With this approach we can consider the properties of electric circuits involving graphene or Weyl semimetals. The existence of the induced chiral magnetic current in a graphene subjected to magnetic field causes an interesting and unusual behavior of such circuits. We discuss an explicit example of a circuit involving the current generation in a "chiral battery". The special properties of this circuit may be utilized for creating "chiral electronic" devices.
\end{abstract}

Keywords: Chiral Dirac; Battery; Grapheme; Weyl Semimetal

\section{Introduction}

Recently, the 2D and 3D materials with linearly dispersing excitations [1] have attracted significant attention. The existence of these "chiral" excitations stems from the point touchings of conduction and valence bands. The corresponding dynamics is described by the Hamiltonian $H= \pm v_{F} \hat{\sigma} \cdot k$; where $v_{F}$ is the Fermi velocity of the quasi-particle, $k$ is the momentum in the first Brillouin zone, and $\hat{\sigma}$ are the Pauli matrices. This Hamiltonian describes massless particles with positive or negative (depending on the sign) chiralities, e.g. neutrinos, and the corresponding wave equation is known as the Weyl equation - hence the name Weyl semimetal [1]. Weyl semimetals are closely related to $2 \mathrm{D}$ graphene [2], and to the topological insulators [3]-3D materials with a gapped bulk and a surface supporting chiral excitations. Specific realizations of Weyl semimetals have been proposed, including a multilayer structure composed of identical thin films of a magnetically doped 3D topological insulator, separated by ordinary-insulator spacer layers [4].

Weyl semimetals provide a unique opportunity to study the macroscopic behavior of systems composed by chiral fermions. In particular, they allow [5] to study, in a condensed matter system, the chiral magnetic effect expected [6-11], and closely related phenomena [12-16]. The effects of the anomaly on the transport in Weyl semimetals, including the chiral magnetic effect, have recently been investigated in [17-20].

In this paper we argue that the existence of chiral magnetic current in graphene subjected to magnetic field can cause an interesting, and potentially useful for practical applications, behavior of circuits such a chiral battery [9].

The material called graphene which is a single layer of atoms arranged in honeycomb lattice could let electronics to process information and produce radio transmission 10 times better than silicon based devices.

From the point of view of its electronic properties, graphene is a two-dimensional zero-gap semiconductor with the cone energy spectrum, and its low-energy quasiparticles are formally described by the Dirac-like Hamiltonian $[21,22] . H_{0}=-i \hbar v_{F} \hat{\sigma} \cdot \nabla$ where $v_{F} \approx 10^{6} \mathrm{~ms}^{-1}$ is the Fermi velocity and $\hat{\sigma}=\left(\sigma_{x}, \sigma_{y}\right)$ are the Pauli matrices. The fact that charge carriers in graphene are described by the Dirac-like Equation (1), rather than the usual Schrödinger equation, can be seen as a consequence of graphene's crystal structure, which consists of two equivalent carbon sublattices [21,22]. Quantum mechanical hopping between the sublattices leads to the formation of two cosine-like energy bands, and their intersection near the edges of the Brillouin zone yields the conical energy spectrum. As a result, quasiparticles in graphene exhibit the linear dispersion relation

$E_{G}=E=\hbar k v_{F}$, as if they were massless relativistic particles with momentum $k$ (for example, photons) but the 
role of the speed of light is played here by the Fermi velocity $v_{F} \approx c / 300$. Owing to the linear spectrum, it is expected that graphene's quasiparticles will behave differently from those in conventional metals and semiconductors where the energy spectrum can be approximated by a parabolic (free-electron-like) dispersion relation.

The low-energy excitations of this system are then described by the massless two-dimensional Weyl-Dirac equation and their energy dispersion relation $\omega=v_{F} k$ is that of relativistic massless fermions with particle-hole symmetry. The maths is simple but the principles are deep. We will review the formulation of graphene's massless Dirac Hamiltonian, under the chiral electromagnetism approach, like a meta-material media [23-29], hopefully demystifying the material's unusual chiral, relativistic, effective theory. These results are derived of the Chiral Electrodynamics with $T$ as the chiral parameter and $k_{0}=\omega / c$ [27,30-32]. The chiral vector potential $A_{c}$ can be expressed as

$$
\nabla^{2} A_{c}+\frac{k_{0}^{2}}{1-k_{0}^{2} T^{2}} A_{c}+2 \frac{\omega^{2} \mu \varepsilon T}{1-k_{o}^{2} T^{2}}\left(\nabla \times A_{c}\right)=0
$$

As $k=\sqrt{k_{x}^{2}+k_{y}^{2}+k_{z}^{2}}$, if $k_{x}=k \sin \theta, k_{y}=0$, and $k_{z}=k \cos \theta$, we have the matrix: (see the formula below)

The dispersion relation of the transversal wave is

$$
\begin{gathered}
\left(-k^{2}\left(1-k_{0}^{2} T^{2}\right)+k_{0}^{2}\right)^{2}-4 k_{0}^{4} k^{2} T^{2}\left(\sin ^{2} \theta+\cos ^{2} \theta\right)=0 \\
\Rightarrow k=k_{ \pm}= \pm k_{0} /\left(1 \pm k_{0} T\right) .
\end{gathered}
$$

That is

$$
k_{ \pm}= \pm k_{0} /\left(1 \pm k_{0} T\right)= \pm \frac{\omega}{c}\left(1 \pm k_{0} T\right)^{-1}
$$

If we put $v_{F}=c\left(1 \pm k_{0} T\right)$ then $v_{F} / c=\alpha$ which is the fine structure constant. Here we have that $A_{c}\|E\| H$, that is the electric field $E$ is quasi parallel to the magnetic wave $H$. The novel result here is that in our chiral theory we do not make $c \rightarrow v_{F}$ but we obtain $v_{F}$ as $v_{F}=c\left(1-k_{0} T\right)$ if $k_{0} T>0$ or $v_{F}=c\left(1 \pm k_{0} T\right)$ if $k_{0} T<0$. In this section we have given an approach of the chiral electromagnetism applied to grapheme. In Section 2, we discuss the two component equations of Dirac electron in grapheme. Section 3 describes a graphene battery device.

\section{Two Component Equations of Dirac Electron in Graphene}

The usual choice of an orthogonal set of four plane-wave solutions of the free-particle Dirac equation does not lend itself readily to direct and complete physical interpretation except in low energy approximation. A different choice of solutions can be made which yields a direct physical interpretation at all energies. Besides the separation of positive and negative energy states there is a further separation of states for which the spin is respectively parallel or anti-parallel to the direction of the momentum vector. This can be obtained from the Maxwell's equation without charges and current in the wave $E \| H$ configuration, so $E=i \eta H$, or $E=i c B$ [30-32]. Here we considerer a bidimensional graphene system so the Dirac's four-component equation for the "relativistic" electron is:

$$
i \hbar \frac{\partial}{\partial t} \psi=\hat{H}^{D} \psi
$$

where $\psi=\left(\psi_{E}, \psi_{H}\right)^{t}$ with $\psi_{E}=\hat{\sigma} \cdot \boldsymbol{E}, \psi_{H}=\hat{\sigma} \cdot \boldsymbol{H}$ so we can write

$$
\begin{gathered}
\hat{H}^{D}=v_{F}(\boldsymbol{\alpha} \cdot \hat{\boldsymbol{p}})+m v_{F}^{2} \beta, \\
\alpha_{k}=\left(\begin{array}{cc}
0 & \sigma_{k} \\
\sigma_{k} & 0
\end{array}\right), \quad k=1,2,3, \\
\beta=\left(\begin{array}{cc}
I & 0 \\
0 & -I
\end{array}\right)=\sigma_{z}
\end{gathered}
$$

and $I$ is the two-by-two identity matrix and $\alpha_{k}$ and $\beta$ are written in chiral or Weyl representation, the Fermi velocity $v_{F}$ is deduced from the chiral electrodynamics with $v_{F}=c\left(1 \pm k_{0} T\right)$, where $T$ is the chiral parameter in a metamaterial condition. This result is capital to our approach because we find a contact point between the graphene system and optical metamaterial making $v_{F}=c\left(1 \pm k_{0} T\right)$, no making $c \rightarrow v_{F}$ as other authors do it. This Hamiltonian commutes with the momentum vector $p$. In order to resolve this degeneracy we seek a dynamical variable which commutes with both $H$ and $p$. Such a variable is $\hat{\sigma} \cdot p$, where $\hat{\sigma}$ is the matrix Pauli. The eigenfunctions of the commuting variables $p$ and $\hat{\sigma} \cdot p$ are simultaneous:

$$
(\hat{\sigma} \cdot p)^{2}=p^{2}
$$

Thus for a simultaneous eigenstate of $p$ and $\hat{\sigma} \cdot p$, the value of $\hat{\sigma} \cdot p$ will be $+p$ or $-p$, corresponding to states for which the spin is parallel or antiparallel, respectively, to the momentum vector like a graphene system.

$$
\left(\begin{array}{ccc}
-k^{2}\left(1-k_{0}^{2} T^{2}\right)+k_{0}^{2} & -2 j k_{0}^{2} k T \cos \theta & 0 \\
2 j k_{0}^{2} k T \cos \theta & -k^{2}\left(1-k_{0}^{2} T^{2}\right)+k_{0}^{2} & -2 j k_{0}^{2} k T \sin \theta \\
0 & 2 j k_{0}^{2} k T \sin \theta & -k^{2}\left(1-k_{0}^{2} T^{2}\right)+k_{0}^{2}
\end{array}\right) \times\left(\begin{array}{c}
A_{x} \\
A_{y} \\
A_{z}
\end{array}\right)=0
$$


A simultaneous eigenfunction of $H$ and $p$ will have the form of a plane wave

$$
\psi_{j}=u_{j} \exp [i(p \cdot r-E t) / \hbar], j=1,2,3,4,
$$

where the $\psi_{j}$ are the four components of the state function and $u_{j}$ four numbers to be determined. Then $E$ can have either of the two values.

$$
E= \pm \varepsilon= \pm\left(m^{2} v_{F}^{4}+v_{F}^{2} p^{2}\right)^{\frac{1}{2}} .
$$

We now demand that $\psi_{j}$ be also an eigenfunction of $\hat{\sigma} \cdot p$ belonging to one of the eigenvalues $p_{\mathrm{E}}$, say, where $p_{\mathbf{E}}= \pm p$, The eigenvalue equation is

$$
\hat{\sigma} \cdot p \psi=p_{\mathbf{E}} \psi,
$$

Since $W$ can be given either of the two values $\pm \varepsilon$ and $p_{\mathrm{E}}$, the two values $\pm p$, we have found for given $p$ four linearly independent plane wave solutions. It is easily verified that they are mutually orthogonal.

The physical interpretation of the solutions is now clear. Each solution represents a homogeneous beam of particles of definite momentum $p$, of definite energy, either $\pm \varepsilon$, and with the spin polarized either parallel or anti-parallel to the direction of propagation. From here we can obtain the well known equation for graphene $H_{0}=-i \hbar v_{F} \hat{\sigma} \cdot \nabla= \pm v_{F} \hat{\sigma} \cdot p$. The sign \pm correspond to the different chiralities of the Weyl fermions. When we apply a magnetic field $B_{0}$ along $z$, we obtain

$H_{0}= \pm v_{F} \hat{\sigma} \cdot\left(p-\mathrm{e} / c A_{0}\right)$, where $A_{0}$ is the vector potential corresponding to $B_{0}$. The chiral anomaly occurs when the current density in terms of right and left-handed spinors is nonzero.

$j^{\mu}=\mathrm{e}\left(\psi_{E}^{\dagger} \sigma^{\mu} \psi_{E}\right)+\mathrm{e}\left(\psi_{H}^{\dagger} \sigma^{\mu} \psi_{H}\right)$ and the total chiral current is

$$
I^{c}=\int j^{\mu} \mathrm{d}^{3} x
$$

In Section 3, we extend this study to 3-D graphene with linearly dispersing excitations envolving an interesting application of the Chiral Magnetic Effect-a rechargeable battery which stores chirality - the chiral battery.

\section{Chiral Current in a Graphene Battery Device}

Chirality in graphene is not related to the usual spin states considered above but instead refers to the sublattice states. If we have some finite amount of this material, it can be used as a battery. The battery can be charged using the axial anomaly by placing it in parallel electric and magnetic fields. The charging time will be determined by the axial anomaly. The battery stores energy, since the Fermi-levels of right- and left-handed modes differ.

In the absence of electric and magnetic fields, chirality is conserved, so the battery does not discharge. If the battery is connected to a circuit element with resistance $R$ and we apply a magnetic field to the battery in the right direction, a current $J$ will be induced due to the Chiral Magnetic Effect. The behavior of this current as a function of the applied magnetic field can be obtained. The current will cause a potential difference $V=I_{\text {total }} R$ over the circuit element. As a result, the same potential difference will also exist over the battery. Hence an electric field will arise parallel to the magnetic field. In this case the axial anomaly operates again to decrease the chirality. Hence a slow rate of discharge will be determined by the axial anomaly as well.

Following the argument by Nielsen and Ninomiya, [17] let us imagine that there is an electric field $E_{0}$ applied in the same direction as the magnetic field $B_{0}$. This $E_{0}$ will lead to transfer of particles between the two Weyl nodes of graphene through the zero node, which has a definite chirality. The slow rate of particles between the left $(-)$ and right $(+)$ node per unit volume is given by

$$
\frac{\mathrm{d}\left(N_{-}-N_{+}\right)}{\mathrm{d} t}=\frac{\mathrm{e}^{2}}{2 \pi^{2} \hbar^{2} c} E_{0} B_{0}
$$

Since the Weyl nodes have an energy difference $\Delta \varepsilon_{W}$, this particle transfer process has an associated power per unit volume

$$
P=\Delta \varepsilon_{W} \frac{\mathrm{d}\left(N_{-}-N_{+}\right) / 2}{\mathrm{~d} t}
$$

So that $P=J E_{0}$ and $J$ can be obtained. Consider first a cylindrical sample of graphene inside a solenoid that provides an external, constant magnetic field of strength $B$ along the longitudinal direction of cylindrical geometry, say $z$, see Figure 1. The top and bottom of the graphene material are touching metallic plates that can conduct electric currents owing through the sample. These two metallic plates are then connected to an outside circuit which is characterized by a resistance $R$. The cross section area of the graphene sample is $\pi r^{2}$ and the

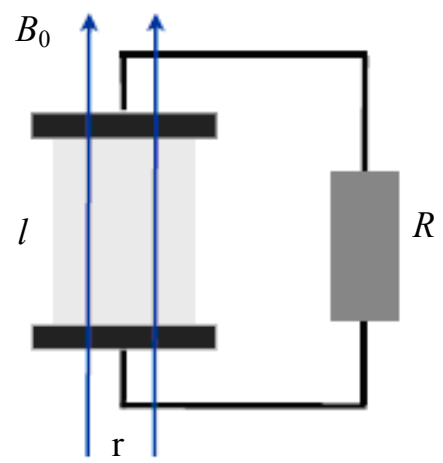

Figure 1. The chiral battery: Graphene system (shown in grey) connected to the circuit with resistance $R$ in an external magnetic field $\boldsymbol{B}_{0}$. 
longitudinal length is $d$. The induced chiral magnetic current density along $z$, is (Equation (9))

$$
J_{c}=\frac{\mathrm{e}^{2} \Delta \varepsilon}{4 \pi^{2} \hbar c} B
$$

$\Delta \varepsilon$ is the energy separation between the Weyl nodes. If $\Delta \varepsilon=1 \mathrm{meV}$, and the magnetic field $B_{0} \sim 1 \mathrm{~T}$, we have $J_{c} \sim 0.1 \mathrm{~A} / \mathrm{cm}^{2}$, an easily measurable current [9].

In the case of a global equilibrium all the contribution to Equation (9) from different valleys cancel each other. In the presence of $B_{0}$ parallel to $E_{0}$, an imbalance of electron populations is created so there is a finite current and it responds only on the component of the electric field parallel to $B_{0}$.

Once an external magnetic field is applied, the energy stored in the difference of the chemical potentials of leftand right-handed fermions can be released by generating the current $\left(I_{c}\right)$, hence the name chiral battery [9].

Note that according to (9) a Weyl semimetal is a kind of battery that provides a definite amount of current, contrary to conventional batteries that support a definite voltage. The total anomaly-induced current through the sample is $I_{c}=\pi r^{2} J_{c}$. If the entire current is $I_{\text {total }}=I_{t}$, then there is a voltage drop along the resistance $R$ given by $V=I_{\text {total }} R$. Since the same amount of voltage drop should also occur along the graphene sample, there is an electric field along $z$ direction with a magnitude

$$
E_{0}=-V / \mathrm{d}=-I_{t} R / \mathrm{d}
$$

Note the negative sign of $E$. This electric field gives rise to a normal current through conductivity $\sigma$

$$
I_{j}=\pi r^{2} \sigma E_{0}=-\frac{\pi r^{2} \sigma R}{\mathrm{~d}} I_{\text {total }}=\frac{R}{R_{j}} I_{\text {total }} ;
$$

where $R_{j}=\frac{\mathrm{d}}{\sigma \pi r^{2}}$

is the intrinsic resistance of the Weyl semimetal sample. The total current $I_{t}$ should be the sum of $I_{j}$ and $I_{c}$, that is determined self-consistently as

$$
I_{t}=I_{j}+I_{c}=\frac{I_{c}}{1+R / R_{j}}
$$

This is the equation governing the performance of the chiral battery.

Let us now see how the energy discharge works for the chiral battery. From the total current (12) through the resistance $R$ and the normal current $I_{j}$. In through $R_{j}$, the energy discharge rate should be

$$
\frac{\mathrm{d} \varepsilon}{\mathrm{d} t}=R I_{t}^{2}+R_{j} I_{j}^{2}=\frac{R}{1+R / R_{j}} A^{2}\left(\frac{\mathrm{e}^{2}}{4 \pi^{2} \hbar^{2} c}\right)^{2} B^{2}
$$

using (11) and (12). This should match the reduction of internal energy of the Weyl semimetal sample. In the presence of both electric field $E$ as in (10) and the mag- netic field $\mathbf{B}$, the charge density of $i$ th Weyl point changes is $(\hbar, c \rightarrow 1)$

$$
\frac{\mathrm{d} \rho_{i}(t)}{\mathrm{d} t}=\frac{k_{i} \mathrm{e}^{2}}{4 \pi^{2}} \boldsymbol{E} \cdot \boldsymbol{B}=\frac{k_{i} \mathrm{e}^{2}}{4 \pi^{2}}\left(\boldsymbol{E}_{0} \cdot \boldsymbol{B}_{0}+\boldsymbol{E}_{w} \cdot \boldsymbol{B}_{w}\right)
$$

Here as the fast time electromagnetic field is $\boldsymbol{E}_{w}=i c \boldsymbol{B}_{w}$, there is not contribution of this term, so the slow time average is

$$
\left\langle\frac{\mathrm{d} \rho_{i}(t)}{\mathrm{d} t}\right\rangle=-\frac{k_{i} \mathrm{e}^{2}}{4 \pi^{2}} \frac{I_{t}}{\mathrm{~d}} B_{0}
$$

The total volume of the sample is Ad, so that the slow total rate of increase of $i$ 'th charge is

$$
\frac{\mathrm{d} Q_{i}}{\mathrm{~d} t}=-\frac{k_{i} \mathrm{e}^{2}}{4 \pi^{2}} A I_{\text {total }} R B_{0}
$$

from which the rate of internal energy change is

$$
\frac{\mathrm{d} \varepsilon_{\text {int }}}{\mathrm{d} t}=-J_{c} A I_{\text {total }} R=\frac{R}{1+R / R_{j}} A^{2}\left(\frac{\mathrm{e}^{2}}{4 \pi^{2} \hbar^{2} c}\right)^{2} B_{0}^{2}
$$

Using the expression (12) for I, which indeed agrees precisely with (13), the time-dependence of the chiral battery performance relies on the detailed equation of state between $\rho_{i}$ and $\mu_{i}$, (chiral chemical potential).

Let us estimate the amount of energy $E$ stored in the chiral battery per unit volume. It is equal to the Helmholtz free energy, which is the energy that can be used to do work. The free energy is the difference between the thermodynamic potential with a chiral charge density and without and is easily founded. Following [9], we then obtain that the energy density is

$$
\frac{\mathrm{d} E}{\mathrm{~d} v o l} \approx 7.0 \times 10^{4} \frac{v_{F}}{c} \mathrm{e} v / \mathrm{nm}^{3} \approx 1.0 \times 10^{7} \frac{v_{F}}{c} \mathrm{~J} / \mathrm{cm}^{3}
$$

The typical distance between the lattice sites in a crystal is of order $0.1 \mathrm{~nm}$. Suppose we can store 1 unit of chirality per lattice site, i.e. an excess of 100 righthanded fermions over left-handed fermions per $\mathrm{nm}^{3}$. In typical materials like graphene $v_{F} / c \approx 10^{-2}$, so the typical storage capacity of the chiral battery is of order $10^{5} \mathrm{~J} / \mathrm{cm}^{3} \approx 30 \mathrm{Wh} / \mathrm{cm}^{3}$. This is comparable or better than conventional batteries whose energy density is typically $10-100 \mathrm{Wh} / \mathrm{Kg}$; note besides that the current in our case is spin-polarized and so may be used for spintronic applications.

The circuit discussed above represents only a couple of examples from a vast array of devices that one can envision. We hope that the chiral electronics based on graphene circuits can serve as a new way to explore the macroscopic dynamics induced by the chiral anomaly, and perhaps open a path towards new electronic devices.

\section{Conclusions}

In this paper, we reviewed the formulation of graphene's 
massless Dirac equation, under the chiral electromagnetism approach. In Dirac's theory, many authors replace the speed of light by the Fermi velocity, in this paper we showed that in graphene the Fermi velocity is obtained from the connection between the electromagnetic chirality and the fine structure constant $v_{F} / c=\alpha$ when the electric wave $\boldsymbol{E}$ is quasiparallel to the magnetic wave $\boldsymbol{H}$. With this approach we considered the properties of electric circuits involving graphene. The existence of the induced chiral magnetic current in a graphene subjected to magnetic field causes an interesting and unusual behavior of such circuits. We discuss an explicit example of a circuit involving the chiral current in a "graphene battery". The special properties of this circuit may be utilized for creating other "chiral electronic" devices.

\section{Acknowledgements}

I wish to thank my colleague Ricardo Ovalle (Escuela de Ingeniería Electrica Electrónica, EIEE), for many useful discussions on batteries.

\section{REFERENCES}

[1] X. Wan, et al., "Topological Semimetal and Fermi-Arc Surface States in the Electronic Structure of Pyrochlore Iridates," Physical Review B, Vol. 83, No. 20, 2011, Article ID: 205101.

[2] A. K. Geim and K. S. Novoselov, "The Rise of Graphene," Nature Materials, Vol. 6, 2007, pp. 183-191.

[3] C. L. Kane and E. J. Mele, " $Z_{2}$ Topological Order and the Quantum Spin Hall Effect," Physical Review Letters, Vol. 95, No. 14, 2005, Article ID: 146802.

[4] A. A. Burkov and L. Balents, "Weyl Semimetal in a Topological Insulator Multilayer," Physical Review Letters, Vol. 107, No. 12, 2011, pp. 127205-127209.

[5] A. A. Zyuzin, S. Wu and A. A. Burkov, "Weyl Semimetal with Broken Time Reversal and Inversion Symmetries," Physical Review B, Vol. 85, No. 16, 2012, pp. 165110165119.

[6] D. Kharzeev and H. Warringa, "Chiral Magnetic Conductivity," Physical Review D, Vol. 80, No. 3, 2009, pp. 34028-34038

[7] D. Kharzeev and A. Zhitnitsky, "Charge Separation Induced by P-Odd Bubbles in QCD Matter," Nuclear Physical A, Vol. 797, No. 1-2, 2007, pp. 67-79.

[8] D. E. Kharzeev and D. Son, "Testing the Chiral Magnetic and Chiral Vortical Effects in Heavy Ion Collisions," Physical Review Letters, Vol. 106, No. 6, 2011, pp. 062301062305. doi:10.1103/PhysRevLett.106.062301

[9] K. Fukushima, D. E. Kharzeev and H. J. Warringa, "The Chiral Magnetic Effect," Physical Review D, Vol. 78, No. 7, 2008, p. 074033. doi:10.1103/PhysRevD.78.074033

[10] D. E. Kharzeev, "Topologically Induced Local P and CP Violation in QCD x QED," Annals Physics, Vol. 325, 2010, pp. 205-229.

[11] B. I. Abelev, et al., "Azimuthal Charged-Particle Cor- relations and Possible Local Strong Parity Violation," Physical Review Letters, Vol. 103, 2009, pp. 251601251605.

[12] A. Vilenkin, "Holographic Multiverse and the Measure Problem," JCAP, Vol. 6, No. 6, 2011, p. 32.

[13] G. M. Eliashberg, "Electrical Current and Magnetic Fields in Conductors with Mirror-Isomeric Structure," JETP Letters, Vol. 38, No. 4, 1983, pp. 220-223.

[14] L. S. Levitov, Y. V. Nazarov and G. M. Eliashberg, "Electron Spin Susceptibility of Superconductors," JETP Letters, Vol. 41, No. 5, 1985, pp. 228-231.

[15] M. Giovannini and M. E. Shaposhnikov, "Primordial Magnetic Fields, Anomalous Isocurvature Fluctuations and Big Bang Nucleosynthesis," Physical Review Letters, Vol. 80, 1998, pp. 22-25.

[16] A. Y. Alekseev, V. V. Cheianov and J. Frolich, "Universality of Transport Properties in Equilibrium, the Goldstone Theorem, and Chiral Anomaly," Physical Review Letters, Vol. 81, 1998, pp. 3503-3506.

[17] H. B. Nielsen and M. Ninomiya, "Physical Derivation of Chiral Anomaly by Using Dirac Sea," Physical Review Letters, Vol. 130, 1983, pp. 389-393.

[18] I. Zahed, “Anomalous Chiral Fermi Surface," Physical Review Letters, Vol. 109, No. 9, 2012, pp. 091603-091607.

[19] D. T. Son and B. Z. Spivak, "Chiral Anomaly and Classical Negative Magnetoresistance of Weyl Metals," 2012.

[20] A. A. Zyuzin and A. A. Burkov, "Topological Response in Weyl Semimetals and the Chiral Anomaly," Physical Review B, Vol. 6, No. 11, 2012, pp. 115-133.

[21] K. S. Novoselov, et al., "Two-Dimensional Gas of Massless Dirac Fermions in Graphene," Nature, Vol. 438, 2005, pp. 197-200.

[22] M. I. Katsnelson, et al., "Chiral Tunnelling and the Klein Paradox in Graphene," Nature Physics, Vol. 2, 2006, pp. 620-624.

[23] R. A. Shelby, et al., "Experimental Verification of a Negative Index of Refraction," Science, Vol. 292, No. 6, 2001, pp. 77-79. doi:10.1126/science. 1058847

[24] V. G. Veselago, "The Electrodynamics of Substances with Simultaneously Negative Values of $\varepsilon$ and $\mu$," Soviet Physics Uspekhi, Vol. 10, No. 4, 1968, pp. 509- 514. doi:10.1070/PU1968v010n04ABEH003699

[25] D. R. Smith, et al., "Composite Medium with Simultaneously Negative Permeability and Permittivity," Physical Review Letters, Vol. 84, No. 18, 2000, pp. 4184-4187. doi:10.1103/PhysRevLett.84.4184

[26] C. Monzon and D. W. Forester, "Negative Refraction and Focusing of Circularly Polarized Waves in Optically Active Media," Physical Review Letters, Vol. 95, 2005, pp. 123904-123907.

[27] H. Torres-Silva et al., "Chiral Waves in a Metamaterial Medium," International Journal of Pure and Applied Sciences and Technology, Vol. 2, No. 2, 2011, pp. 54-65.

[28] E. Plum, J. Zhou, J. Dong, V. A. Fedotov, T. Koschny, C. M. Soukolis and N. I. Zheledev, "Metamaterial with Negative Index Due to Chirality," Physical Review B, Vol. 79, No. 3, 2009, pp. 0354071-0354076. 
doi:10.1103/PhysRevB.79.035407

[29] J. Zhou, J. Dong, B. Wang, T. Koschny, M. Kafesaki and C. Soukoulis, "Negative Refractive Index Due to Chirality," Physical Review B, Vol. 79, 2009, pp. 121104-121107.

[30] H. Torres-Silva, "The Close Relation between the Maxwell System and the Dirac Equation When the Electric Field Is Parallel to the Magnetic Field," Ingeniare, Vol. 16, No. 1, 2008, pp. 43-47.

[31] H. Torres-Silva, "Dirac Matrices in Chiral Representation and the Connection with the Electric Field Parallel to the Magnetic Field," Ingeniare, Vol. 16, No. 1, 2008, pp. 4852.

[32] H. Torres-Silva, "Chiral Transverse Electromagnetic Standing Waves with E II $\mathrm{H}$ in the Dirac Equation and the Spectra of the Hydrogen Atom," In: A. Akdagli, Ed., Behavior of Electromagnetic Waves in Different Media and Structures, Intech, Croatia, 2011, pp. 301-324. 